\title{
Artificial Intelligence Applications in Power System
}

\author{
Xiaoyue Zhao and Xinyan Zhang* \\ Xinjiang University, China \\ ${ }^{*}$ Corresponding author
}

\begin{abstract}
The purpose of this paper is to research on artificial intelligence in power system operation and on transient protection, through the use of discrete control and the continuous control to increase the stability of the power system; transient protection is through the use of relay protection based on expert system and fuzzy logic in the protection setting calculation of expert and fault diagnosis, greatly improved the efficiency of solving the problem. This paper originally were studied in artificial intelligence in power system on steady and transient, introducing the most suitable method, which can be used as reference in practical application. All in all artificial intelligence is the ability using computers to perform some complex problems to solve, such as image recognition, judge the ability to understand the problem.
\end{abstract}

Keywords-component; Artificial intelligence; ANN; The theory of fuzzy recognition and diagnosis; Expert system; Genetic algorithm.

\section{AN OVERVIEW OF ARTIFICIAL INTELLIGENCE APPLICATIONS IN POWER SYSTEMS}

\section{A. The Cause of the Use of Artificial Intelligence in Power Systems}

In power system operation and control the basic goal is to provide users with quality electricity power in economic rational degree for power system, and ensure their stability and reliability. So it is necessary to carry out the planning of power system monitoring and control, but with the development of the electric power system, the demand is coming more and more, in order to ensure its safety, economic and reliable operation of the workload increase, it also has been increased the burden of staff operation. The computer software of the existing EMS center is usually the numerical analysis software, it is difficult to have the target processing in operation of the power system, especially in the fault condition. Using artificial intelligence techniques to assist operational personnel to check and judge greatly reduces the workload of operational staff also improves the efficiency of dealing with incidents. This is one of the main causes in recent years that researches on application of electric power workers poured into artificial intelligence [1].

\section{B. Artificial Intelligence Overview}

Artificial intelligence is a scientific discipline aiming to research, develop and simulate of human behavior and its rules. Artificial intelligence techniques including Brain Science, Neurology, information technology and various discipline artificial intelligence techniques including Brain Science, Neurology, and disciplines such as information technology, widely used in all walks of life, through to mimic the behavior of the human brain, developed a way to replace human brains discover, identify and analyze machine, improve efficiency and save money. Traditional artificial intelligence includes problem solving and in the process of development to improve the AI induction and basic search technology; meanwhile, in logical reasoning and theorem proving, automatic programming, natural language understanding, expert system all get great achievements. But as for improving own learning and performance aspects have no substantive progress. Limitations of traditional artificial intelligence can only simulate one logical thinking to solve the problem, and cannot be solved in addition to logical thinking, dynamic thinking and some problems that cannot be represented into the sequence of symbols. But in recent years, another kind of intelligent way that artificial neural networks develop fast and can be combined with the traditional AI, to make up for the shortcoming of traditional AI. Modern artificial intelligence technologies include:

\section{(1) The artificial neural network}

The artificial neural network(ANN) Artificial neural network (ANN) is from the perspective of simulated neurons process information using nonlinear mapping method of brain information processing, storage and search mechanism and combining it with AI mechanism. Through connections in a number of simple elements and samples to learn, constantly adjusting weight finally getting the right results and resolve the complex equations and nonlinear problems brought by the difficulties. And ANN massively parallel processing capabilities, and ANN's large scale parallel processing ability, adaptive learning ability, using information distributed storage capacity and robustness of fault tolerance and generalization ability in fault diagnosis have important application.

(2) The theory of fuzzy recognition and diagnosis

Fuzzy theory by definition is fuzzy concepts and mathematical models for handling the practical concept is not clear or obscure facts such as: excessive current, too much loss in which membership does not clear. Fuzzy identification on uncertain events and phenomena are screened to establish mathematical model and input variables. The problem and efficiency of fault diagnosis in power system are solved and improved. Fuzzy recognition theory is mainly used in the protection of the main transformer protection, line protection and discovery and protection, and so on [2]. Because the fuzzy system will not determine the problem is not in the pattern of accuracy and will no longer be uncertain factors on the strict requirements of strict matching, so as accurately for approximately to a certain extent, the fault tolerance of expert system is increased. 
At the same time, the accuracy and efficiency of fault diagnosis are improved. Fuzzy integration theory has been widely used in recent years. Fuzzy theory has been widely used in recent years and various aspects of all fields. Used in transmission and distribution power flow state, under different conditions of load and on the user's classifications is important as to construct the protection principle of transformer, looking for External fault such as currents, extreme and current transformer saturation In search of the optimal between solution power system safe operation conditions and transmission capacity improved accuracy.

\section{(3) Expert system}

Expert system is a kind of intelligent computer software system which is built by human experts. It contains a 3large amount of professional knowledge and rich experience in the power system. Its use has penetrated into all fields. Especially in the field of artificial intelligence technology and even exceed the level of human expert. In medical diagnosis, geological exploration, culture and education has been equipped with the corresponding knowledge and procedures of the system and the problem of solving and processing has been close to the level of experts.

\section{(4) Genetic algorithm}

Genetic algorithm simulate of the computer model of evolutionary in biology to natural selection and genetic mechanism. The aim is to find the optimal solution in the large search space, which can be adapted to the transformation between the individual units and the control strategy of the search group, and the algorithm is simple and robustly which can be very good in traditional search methods in solving complex problems and nonlinear problems, used for power generation planning, transmission and distribution system planning, adjusting the parameters of excitation, to solve the control problem of reactive power compensation and voltage.

\section{Development Trend of Artificial Intelligence in Electric Power System}

Artificial intelligence power system is developing rapidly at present, artificial intelligence includes expert system, artificial neural network, fuzzy diagnosis theory and genetic algorithm. Each has its advantages and limitations, and the lack of a universal and effective method applied to all fields of power system. Hybrid intelligence, that is, a variety of intelligent technology, becoming one of the important development direction of AI [3]. Distributed artificial intelligence is a branch of artificial intelligence research, in which parallel distributed computing eventually come into being. Now the structure of the neural network itself and the improvement of the algorithm is also an important task of AI exploration. More classic round cell neural network BP network with bounded generalization and refuse good characteristics.

\section{APPLiCATIONS AND PROTECTIONS OF ARTIFICIAL INTELLIGENCE SYSTEM}

With the rapid development of China's power grid scale and the construction of the special high-voltage power grid in North China, central China and East china. The need to consider the factors that affect the safe and stable operation of power grid more and more, the grid running mechanisms are more complex. In order to study the power system for the use of artificial intelligence tools to solve the problem of the characteristics divided problem on time frame into real-time control, management planning two parts [4].

\section{A. The Application of Artificial Intelligence in the System Operation Control}

Distribution of electric power system automation and manual control devices such as circuit breakers, relays, disconnecting switch and so on. These relatively simple local individuals element control complex and together constitute a whole power system real-time control that is discrete control and continuous control.

\section{- $\quad$ Discrete control}

\section{(1) Cut load}

Load shedding is another kind of discrete control. When the system is disturbed or the generator is shut down, the capacity of the system is changed dramatically, while the load is kept at a high level, which is far beyond the system's supply, not to cause blackouts must reduce the load. Described by a differential problem of load demand and analysis of system behavior to control the relay action in a timely manner, you can use the function map to reflect. Artificial neural networks has the approximation of function mapping functions and parallel processing capabilities, it has a good adaptability to deal with load control and real-time, select an appropriate character input describing function mapping of key issues.

\section{(2) Power system relay protection}

Relay protection is a kind of common discrete control, which is to detect and judge the normal or fault state in the system and can quickly react to the protection action. The ability to respond quickly and deal with the problem of AI has become an important tool for online assessment. Fuzzy identification theory contains a large area in the design of relay protection .Due to the adaptive ability of traditional protection, reaction ability of detection ability is limited, real-time tracking using the computer, the calculation characteristics of the running mode of protection device, making fuzzy in artificial intelligent control theory for the improvement of the computer provides an important method and way to realize the relay protection device.

\section{- Continuous control}

Excitation control is an important part of controlling the voltage and reactive power of the generator. It can maintain the stability of the power system in the disturbance environment. It is an important real time continuous control system against disturbance. But due to the application of large capacity unit input and fast excitation system, the dynamic stability of the system is coming into being, decreased ability of antiinterference, so the fuzzy set theory in the excitation control system greatly increases the excitation control system control rate and increases the stability of the power system. 


\section{B. Application of Artificial Intelligence in Transient Protection}

\section{- Concept of transient protection}

Transient protection through detection of transient high frequency model for power system transmission line and power system to realize its own functions, the fault transient models include a lot of fault type, position, direction, duration, and so on. First of all, we must use the specific high-frequency detection device and algorithm to extract the high frequency signal from the fault model then use the special fast signal processing algorithm to determine what kind of fault. The principle block diagram of transmission line transient protection is shown in Figure 1.

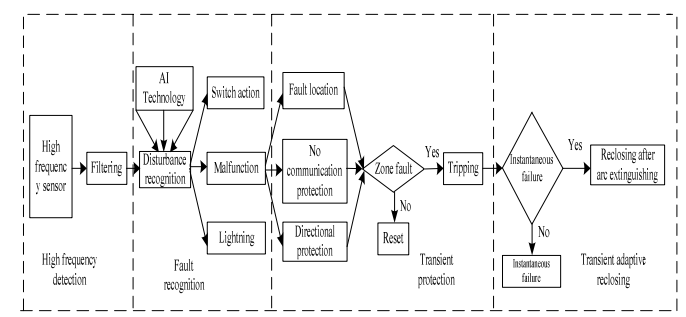

FIGURE I. THE PRINCIPLE BLOCK DIAGRAM OF TRANSMISSION LINE TRANSIENT PROTECTION

In Figure I, the transient protection including high frequency detection, fault identification, transient protection and transient adaptive reclosing these parts, switching in power system fault, lighten can generate high frequency signal, and transmission in the power system, a high frequency detector in the outlet line can detect the identification of non-fault disturbance. When fault happening through fault protection, non-communication protection, transient protection principle to determine whether protection for area fault, if is ,tripping and then enter Adaptive reclosing unit, after tripping in high frequency signal analysis to identify whether the fault is a permanent fault, if it is not to close.

- Application of artificial intelligence in transient protection

The application of artificial intelligence mainly includes the application of expert system, the application of artificial neural network, fuzzy set theory and genetic algorithm. But in the study of transient protection, it is used in the following two ways: the protection of expert system and fuzzy logic protection.

(1) Based on the expert system (system Expert) relay protection.

The expert system has been applied in the power system for many years, but because of the protection of power system has strict requirements on reaction time, so the existing expert system is applied to the time required for protection is not very strict, such as: the choice of protection setting, fault diagnosis and fault location [5]. This paper introduces the expert system used in relay protection setting calculation, through the general rules, comprehensive, comprehensive consideration of relay protection system setting involves the problem, to solve the contradictions of setting conflict.

\section{(2) Fuzzy logic protection.}

The variation of power system load is varied, such as the change of load, the diversification of power system network structure such as high voltage direct transmission, flexible transmission and series capacitor compensation. The relationship between the structure and components of these systems are very complex, which increases the difficulty of protecting, when disturbance occurs because of the above factors, there are too many uncertainties, not well defined and determined, so the input and output in the protection phase and the intermediate model system has fuzziness. It is precisely just because this fuzziness is more close to the actual characteristics, all of the conditions are given to consider more close to the practical application of [6] In this paper, fuzzy method is used to diagnose transformer fault, and the high frequency signal generated by partial discharge and the change of chemical composition in transformer are established. The fuzzy set is established, and the protection of transformer is achieved. But it is difficult to model the fuzzy technology, so it is often combined with neural network, and the neural network is composed of a large number of individual neurons in a certain way. A single neuron is cover input to output transfer function of nonlinear functions, and the combination of a large number of neurons and the achievement is very complex and nonlinear, and carries a lot of weight on the implicit in the information which can be achieved by adjusting the weights of neural network from the complex nonlinear mapping of artificial neural network $\mathrm{m}$ to $\mathrm{N}$ dimensions are widely used for transient protection fault diagnosis and fault line selection [6]. Fast and accurate, and is not affected by the system operating mode, fault type, transformer saturation and other factors; for non communication protection, can extract the fault frequency signal, obtain good simulation results; for lightning wave, identification, switching operation and fault traveling wave is easy to converge.

\section{SUMMARY AND OUTLOOK}

This paper introduces the application of artificial intelligence in power system in recent years, including artificial neural network, expert system, fuzzy theory and so on. These applications can greatly improve the efficiency of the power system, reduce the input of human and material resources, and play an important role in power system security.

In the future, the scale of power system will continue to expand, its complexity is also constantly improved and will bring some more difficult factors to deal with, in which some artificial intelligence currently have their own different advantages and disadvantages and limitations, lacking of a power system applied to the effective hybrid intelligent, namely seek a more suitable method for artificial intelligence processing problems in power system that combines the advantages of artificial intelligence. Believe in the future, with the deepening of research, artificial intelligence will become more and more mature, and easier to be operate, to better solve the problems in power systems. In a short, the integration of a variety with artificial intelligence is a major trend in the future development. 


\section{ACKNOWLEDGMENT}

This work is supported by National Science Foundation of China (51367015)

\section{REFERENCES}

[1] Zhao Gang. Artificial intelligence technology and its application in relay protection [J]. Jiangsu science and technology information, 2014 (24). J. Clerk Maxwell, A Treatise on Electricity and Magnetism, 3rd ed., vol. 2. Oxford: Clarendon, 1892, pp.68-73.

[2] Wang Lu, Mou Jiaqi, Hou Jianping, et al. Research progress on electrode selection for hydrogen production from water electrolysis [J]. chemical industry development, 2009, 28 (S1):512-515.

[3] Tang Huajin, Chen Hanping. Research on the application of artificial intelligence technology in electric power system [J]. electric power construction, 2002, 23 (1):42-44.

[4] Power system relaying committee mark enns et al.Working group D10 of the line protection subcommittee.Potential applications of expert systems to power system protection.IEEE Trans on Power Dalivery,1994,9(2):720 728

[5] Lee S J.An expert system for protective relay setting of transmission systems.IEEE PWRD,1990,5(2):705 714Y.

[6] Liu Lifeng, high school and Germany. Fuzzy knowledge processing of power system relay protection expert system. Automation of electric power systems, 1997,21 (6): $34 \sim 37$ proposed the knowledge expression method and its reasoning model of relay protection fuzzy design. 